\title{
Kinematic considerations for mantle mixing
}

\author{
Guy Metcalfe', Craig R. Bina ${ }^{2}$, and J. M. Ottino ${ }^{1}$
}

tal and computational mixing studies in highly controlled flows show that even seemingly inconsequential errors in the computed velocity field of 3-D or time-dependent flows can cause enormous changes in the advection patterns. These velocity field errors can arise from numerical sources and, more importantly, from imperfect rheological models. The results obtained to date are based on idealized flows, but their very simplicity makes them amenable to both in-depth theoretical study and a considerable degree of experimental investigation [Ottino et al., 1988, 1992; Ottino, 1989, 1990a, 1990b; Jana et al., 1994].

\section{Computations}

The fact that a flow is chaotic greatly accelerates the loss of accuracy, affecting not only the useful length of simulations, but also what quantities can even be meaningfully computed. Extensive computations point out the magnitude of these effects. There are two types of errors to be considered. The first are numerical errors, namely round-off, discretization, and integration errors. The second are model mismatch errors, such as specification of a rheology which does not exactly match that of the physical system. Both error sources are unavoidable in mantle computations. Flows with analytic solutions allow systematic, detailed investigation of the effects of all sources of error.

The most stringent computational test corresponds to an individual particle trajectory. After only 2 or 3 circulation times, an error in the velocity field greater than $\mathcal{O}(0.1 \%)$ produces a deviation of the computed from the true trajectory which is of the order of the size of the domain. If, on the other hand we calculate the advection pattern (large folds, islands, striation patterns, etc.) by starting many points from nearly the same initial location, mimicking a dye advection experiment, velocity field errors of $\mathcal{O}(1 \%)$ produce correct advection patterns: patterns identical to those obtained using analytical velocities and nearly indistinguishable from corresponding experiments. This can be understood by noting that errors in particle positions are along manifolds rather than normal to them, so that numerical trajectories continue to mark the manifolds which are therefore the robust features of chaotic flows. This behavior is a consequence of the sensitivity to initial conditions as expressed by the "shadowing lemma" [Grebogi et al., 1990]. Simply, this lemma states that associated with almost every trajectory in the error-free dynamical system, there is a nearby trajectory in the simulated system that remains close to ("shadows") the actual trajectory for some (accuracy-dependent) duration. Computing many trajectories at once thus produces an accurate picture of the system at large.

Larger velocity field changes, above the $2-3 \%$ level with respect to some reference case, are produced by, for example, rheological or thermal variations. This is of particular 


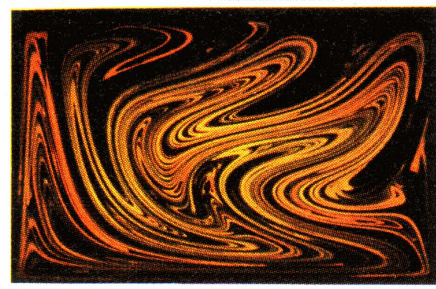

(a)

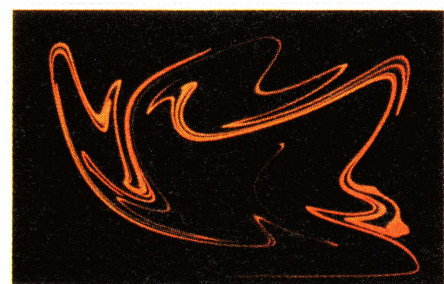

(b)
Figure 1. Small changes in rheology cause substantial changes in large scale advection patterns. The flow is a 2-D Stokes flow in a box [Leong and Ottino, 1989]. The sidewalls are stationary while the top wall moves left to right and the bottom wall moves right to left. The walls move alternately to create time-dependent chaotically advecting flow. Results after 7 overturn periods for an initially compact dye blob [Niederkorn and Ottino, 1993; Leong and Ottino, 1990]. (a) Newtonian fluid (glycerin). (b) Slightly viscoelastic fluid (125 ppm polyacrylamide in glycerin). Large islands appear in the viscoelastic fluid where the Newtonian fluid is well-mixed.

relevance to mantle simulations for which the exact rheology is unknown. With a simulation the typical (often implicit) assumption is that if a rheological model is "close" in some sense to representing the mantle then the simulation results will be "close" as well. This assumption holds true for velocity profiles or streamlines, but it is definitely not true for the transport and mixing characteristics. This sensitivity of the transport to small changes in the velocity field is intrinsic to the nonlinear advection. To the extent that fully detailed

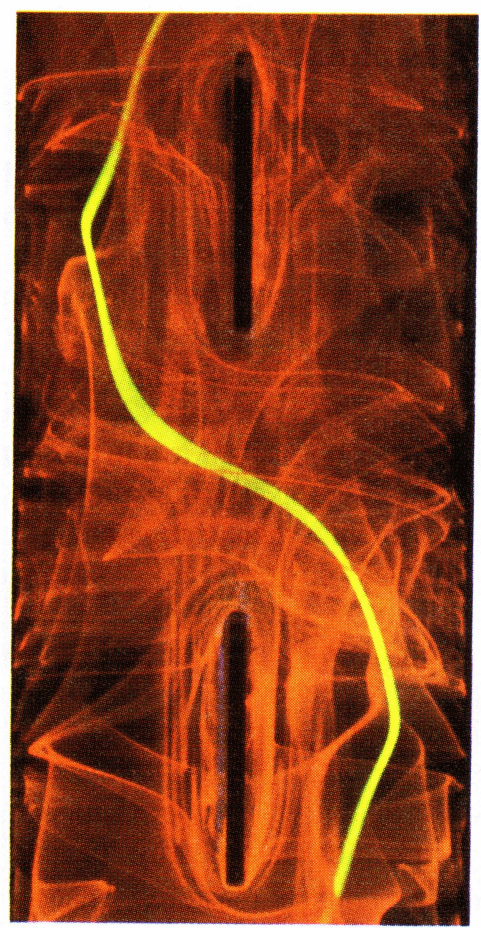

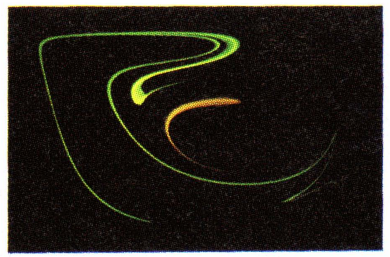

(b)

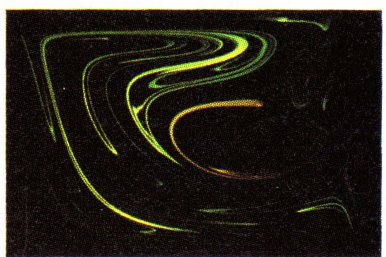

(c)

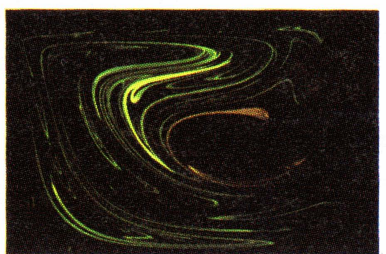

(d)

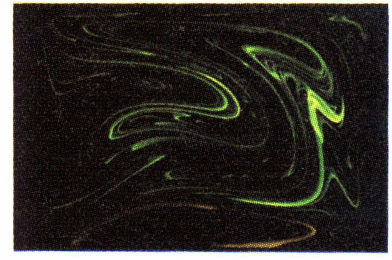

(e)

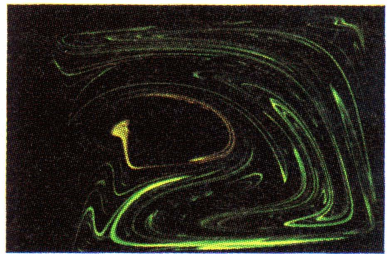
(f)

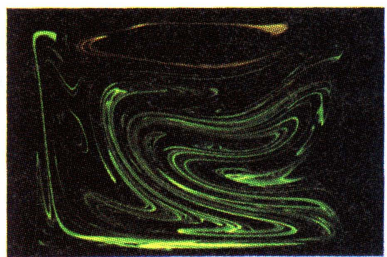

(g)

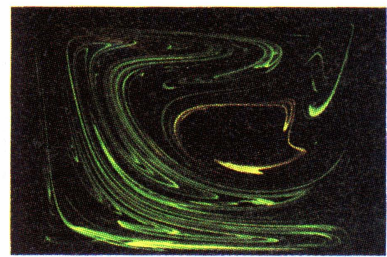

(h) Figure 2. A stable flow tube surrounded by chass in a
section of steady 3-D laboratory flow [Kusch and Ottino, 1992]. Plates arranged periodically in the flow split and reorient the fluid while a circulation is imposed. Material in the tube does not mix while material in the chaotic region is well-mixed.

simulations of the mantle are prohibitive and that parameters only imperfectly known, it should be kept in mind, as evolve smoothly with increased computational realism.

However, this does not imply that nothing can be reliably known. Although it is generally impossible to predict advection patterns in time-dependent flows by an examination are built from only a few general features. Mixing in regular and chaotic regions. Although examples using timeperiodic flows are more numerous, this general structure is so present in aperiodic and even turbulent flows [Elhmaid and chains of islands-stretch, rotate, and contract, but never fields, with islands corresponding to tubes of unmixed fluid shooting through the chaotic region (Figure 2).

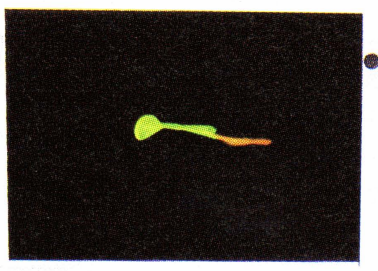

(a)

Figure 3. Chaotic advection in a box of Newtonian fluid with circulating streamlines similar to Figure 1. (a) Initial condition. (b-d) State after 2, 4 and 6 overturn times. (e-h) State after $6.25,6.5,6.75$, and 7 overturn times. 
Since these ideas are purely kinematic in nature, the particular flow is largely irrelevant. Furthermore, the qualitative advection results are independent of how the flow is forced: time-dependence can be induced just as well by boundary motions as by buoyancy or concentration fluxes. In fact any flux that induces a flow and might itself be time-varying [ $\mathrm{Kel}$ logg and Stewart, 1991; Gurnis, 1986] or of such strength as to trigger a time-dependent instability produces the same general picture of regular and chaotic regions in motion. This happens with very simple, even analytically known, velocity fields, yet even so it is impossible to predict or understand the advection patterns by an examination of streamlines without the techniques developed in chaos/mixing theory.

\section{Experiments}

These general kinematic considerations can account quite naturally for certain geochemical observations, such as the persistence of distinct, long-lived geochemical reservoirs at a variety of scales in an actively convecting mantle. Models of layered, convectively isolated reservoirs often have been invoked [Wasserburg and DePaolo, 1979; Anderson, 1984] to explain mixing between layers via ascent of plumes from the transition zone [Irifune and Ringwood, 1993; Ringwood, 1994] or core-mantle boundary [Hart et al., 1992] and/or by some degree of slab penetration into the lower mantle [Silver et al., 1988; Peltier and Solheim, 1992J. However, the geophysical evidence for such compositional layering remains ambiguous [Bina and Silver, 1990]. Other models introduce heterogeneity by subduction, delamination, melting, entrainment, etc., allowing the reservoirs to exist as distributed parcels of material rather than as distinct layers [Gurnis and Davies, 1986; Davies, 1990]. Kellogg [1993] describes such processes in a recent review of mantle mixing.

Experiments (Figure 3 ) in a box of fluid show the coexistence of well-mixed and distinct heterogeneous source regions wilthout invoking special hypotheses. Although laboratory experiments can only caricature mantle flow, plumes are the only qualitative features present in buoyancy driven flows which are missing from our laboratory flows. Figure 3a shows an initial line of the fluid dyed to visualize the subsequent mixing. Figures $3 \mathrm{~b}-\mathrm{d}$ show the mixing after the first three successive overturn times. The orange part of the line falls in an island, where it undergoes only weak rotation; the yellow-green part of the line is in the chaotic region, where it is exponentially stretched. The island appears stationary because pictures are taken at the end of each overturn. During an overturn period, the island moves throughout the domain (Figure $3 \mathrm{e}-\mathrm{h}$ ). The parameters of Figure 3 are in no way spccial: many other levels of encrgy input or flowforcing methods, including non-periodic motions [Elhmaidi et al., 1993; Babiano et al., 1994], produce qualitatively the same picture of well-mixed regions and unmixed reservoirs in motion.

The structure of the dye composition along a fixed line may be measured through digital images similar to those of Figure 3. Consider an observation line fixed between the black dots in Figure 3a. Figure 4a shows the composition (grayscale light intensity from the dye) along this line as a function of overturn times. As time passes, the initial dye streak becomes stretched and folded by the flow so that the
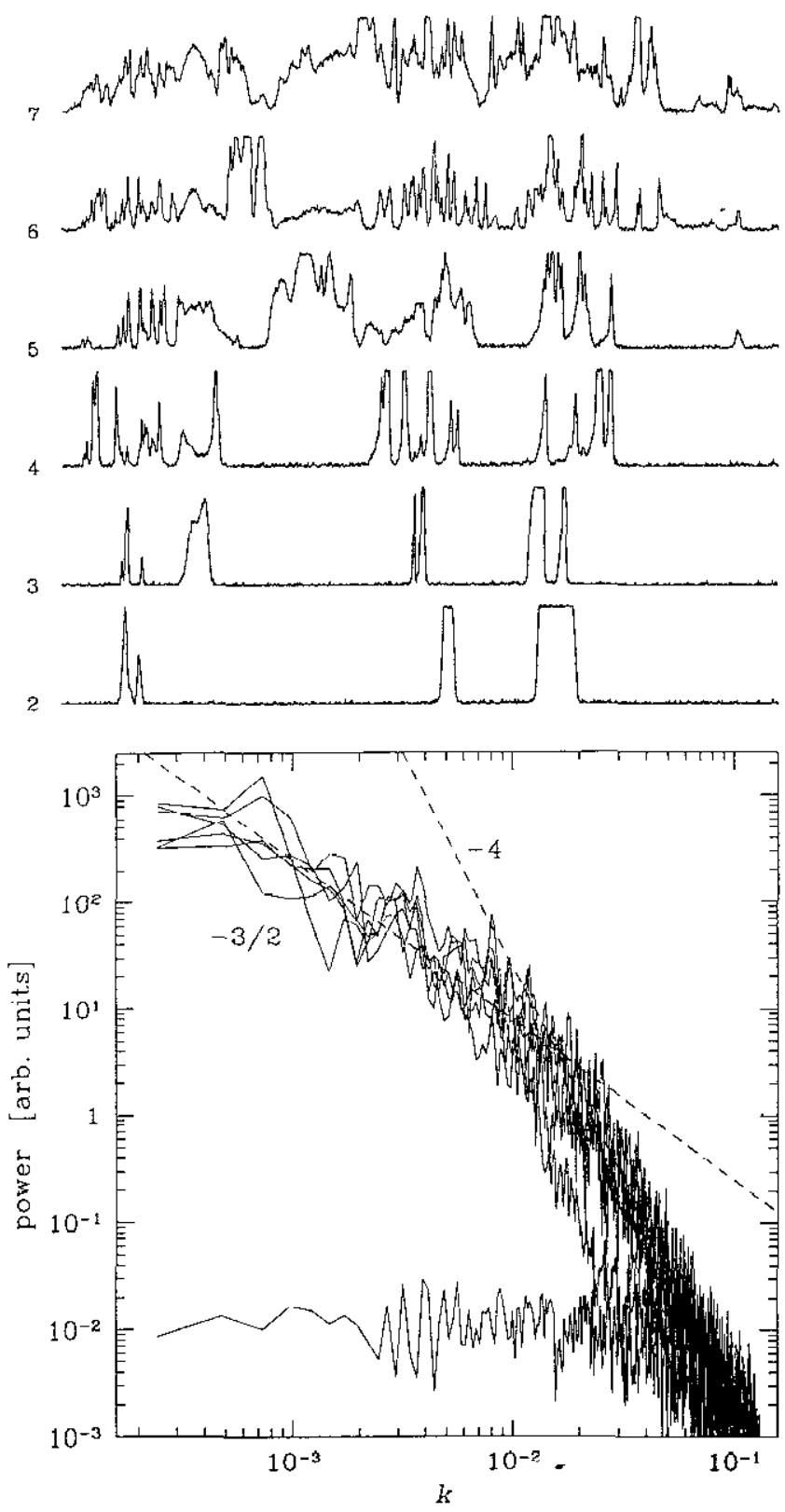

Figure 4. Even if ficld composition measurements were available in laboratory detail, one could still not reliably infer the underlying flow. (a) Dye composition along a line stretched across the box between the black dots in $a$. Overturn times are indicated on the left. The vertical scales in figure $4 \mathrm{a}$ are the same for each graph and indicate grayscale light intensity from the dye. As time passes, the initial dye streak becomes stretched and folded by the flow so that the intersections with a test line become progressively thinner and more numerous. Any number of different flows could produce the same refinement of striations. (b) Fourier spectra of (a). Lengths are scaled so that the box width is unity; the sampling frequency is $1 / 4096$. The line at $10^{-2}$ power shows the measurement noise (i.e. the spectrum for Figure $3 a)$. The spectra are quite similar for all times with two power-law regions. The dotted lines have slopes of $-3 / 2$ and 4.

interscctions with the test line become progressively thinner and more numerous. Figure $4 \mathrm{~b}$ shows the corresponding Fourier transforms of compositional variation along the test line. The different slopes suggest different scaling regimes 
for long- and short-range order. Unfortunately, even if field composition measurements as detailed as those possible in the laboratory were available, one still could not reliably infer the flow that produced the compositional structures: any number of different flows yield the same refinement of striations. What can be said, though, is that if the striation distribution is log-normal, the underlying flow is chaotically advecting [Swanson et al., 1992].

\section{Discussion}

Several rccent developments in mixing theory are relevant to questions arising in studies of mixing and transport in the mantle. Geochemical observations requiring long-term coexistence of isotopically distinct mantle source regions demand no special modeling requirements. Even a steady 3-D flow produces well-mixed and non-mixing regions that preserve long-lived distinct reservoirs, in addition to distributing the regions irregularly in less than ten circulation times. Computations of a chaotic flow requiring accurate individual trajectories are likely to be unreliable, but computations of large scale mixing structures (folds, islands, manifolds, etc.) based on the collective behavior of many trajectories are likely to be reliable. However, advection results do not respond smoothly to added realism in the simulation.

The ideas in this paper were developed using simple and rather restricted example flows. However, the results follow from rather general considerations of the kinematics of fluids and are, in particular, pertinent to mantle flows. Assessment of the ultimate impact of these results upon mantle studies must await their incorporation into future models and simulations.

Acknowledgments. Wc thank Bob Geller, Bill White, and an anonymous reviewer for helpful comments on the manuscript. C.R.B. acknowledges the support of National Science Foundation grant EAR-9158594.

\section{References}

Anderson, D. L., The earth as a planet: Paradigms and paradoxes, Science, 223, 347-355, 1984.

Babiano, A., G. Boffetta, A. Provenzale, and A. Vulpiani, Chaotic advection in point vortex models and $2 \mathrm{~d}$ turbulence, Phys.Fluids, $6,2465-2474,1994$.

Bina, C. R., and P. G. Silver, Constraints on lower mantle composition and temperature from density and bulk sound velocity profiles, Geophys. Res. Lett., 17, 1153-1156, 1990.

Davies, G. F., Mantle plumes, mantle stirring, and hotspot chemistry, Earth Planet. Sci. Lett., 99, 94-109, 1990.

Elhmaidi, D., P. Provenzale, and A. Babiano, Elementary topology of 2-dimensional turbulence from a Lagrangian viewpoint and single-particle dispersion, J. Fluid Mech., 257, 533-558, 1993.

Grebogi, C., S. M. Hammel, J. A. Yorke, and T. Sauer, Shadowing of physical trajectories in chaotic dynamics: Containment and refinement, Phys. Rev. Lett, 65, 1527-1530, 1990.

Gurnis, M., Stirring and mixing in the mantle by plate-scale flow: large persistent blobs and long tendrils coexist, Geo. phys. Res. Lett., 13, 1474-1477, 1986.

Gurnis, M., and G.F. Davies, The effect of depth-dependent viscosity on convective mixing in the mantle and the possible survival of primitive mantle, Geophys. Res. Lett., 13, 541-544, 1986.

Hart, S. R., E. H. Hauri, L. A. Oschmann, and J. A. Whilehead,
Mantle plumes and entrainment: Isotopic evidence,Science, 256 , 517-520, 1992.

Hart, S., and A. Zindler, Constraints on the nature and development of chemical heterogeneities in the mantle, in Mantle Convection: Plate Teclonics and Global Dynamics, pp. 261-387 Gordon and Breach Science Publishing, New York, 1989.

Irifune, T., and A. E. Ringwood, Phase transformations in subducted oceanic crust and buoyancy relationships at depths of $600-800$ $\mathrm{km}$ in the mantle, Earth Planet. Sci.Lett., 117, 101-110, 1993.

Jana, S. C., G. Metcalfe, and J. M. Ottino, Experimental and computational studies of mixing in complex Stokes flows, J. Fluid Mech., 269, 199-246. 1994.

Kellogg, L. H., Chaotic mixing in the earth's mantle, Advan. Geophys., 34, 1-33, 1993.

Kellogg, L. H., and C. A. Stewart, Mixing by chaotic convection in an infinite Prandtl number fluid and implications for mantle convection, Phys. Fluids, A3, 1374-1378, 1991.

Kusch, H. A., and J. M. Ottino, Experiments on mixing in continuous chaotic flows, J. Fluid Mech., 236, 319-348, 1992.

Leong, C.-W., and J. M. Ottino, Experiments on mixing due to chaotic advection in a cavity, J. Fluid Mech., 209, 463-499, 1989.

Leong, C.-W., and J. M. Ottino, Increase in regularity by polymer addition during chaotic mixing in two-dimensional flows, Phys. Rev. Lett, 64, 874-877, 1990.

Niederkorn, T. C., and J. M. Ottino, Mixing of viscoelastic fluids in time-periodic flows, J. Fluid Mech., 256, 243-268, 1993.

Ottino, J. M., The mixing of fluids, Sci. Amer., 260 (1), 56-67, 1989.

Ottino, J. M., The Kinematics of Mixing: Stretching. Chaos, and Transport, 364 pp., Cambridge University Press, Cambridge, England, 1990a.

Ottino, J. M., Mixing, chaotic advection, and turbulence, Ann.Rev. Fluid Mech., 22, 207-254, 1990b.

Ottino, J. M., C.-W. Lcong, H. Rising, and P. D. Swanson, Morphological structures produced by mixing in chaotic flows, Nature, $333,419-425,1988$.

Ottino, J. M., F. I. Muzzio, M. Tjahjadi, J. G. Franjione, S. C. Jana, and H. A. Kusch, Chaos, symmetry, and self-similarity: Exploiting order and disorder in mixing processes, Science, 257, 754-760, 1992.

Peltier, W. R., and L. P. Solheim, Mantle phase transitions and layered chaotic convection, Geophys. Res. Lett., 19, 321-324, 1992.

Ringwood, A. E., Role of the transition zone and $660 \mathrm{~km}$ discontinuity in mantle dynamics, Phys. Earth Planet. Inter., in press, 1994.

Silver, P. G., R. W. Carlson, and P. Olson, Deep slabs, geochemical heterogeneity, and the large-scale structure of mantle convection: Investigation of an enduring paradox, Ann. Rev. Earth Planet. Sci., 16, 477-541, 1988.

Swanson, P. D., F. J. Muzzio, and J. M. Ottino, Mixing distribution produced by multiplicative stretching in chaotic flows, Int. J. Bifurcations and Chaos, 2, 37-50, 1992.

Wasserburg, G. J., and D. J. DePaolo, Models of earth structure inferred from neodymium and strontium isotopic abundances, Proc. Nat. Acad.Sci. USA, 76, 3594-3598, 1979.

C. R. Bina, Department of Geological Sciences, Locy Hall, Northwestern University, Evanston, IL 60208-2150. (e-mail: craig@earth.nwu.edu)

Guy Metcalfe and J. M. Ottino, McCormick School of Engineering and Applied Science, Department of Chemical Engineering, Northwestern University, Evanston IL 60208-3120. (email: guy@chem-eng.nwu.edu, ottino@chem-eng.nwu.edu

(received July 18, 1994; revised October 29, 1994; accepted November 29, 1994.) 